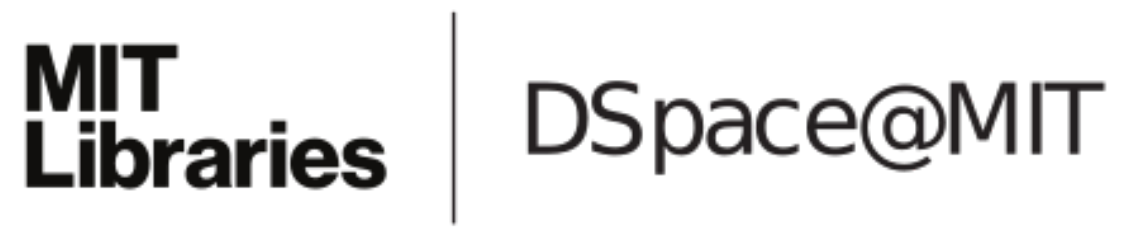

\author{
MIT Open Access Articles
}

Profit loss in Cournot oligopolies

The MIT Faculty has made this article openly available. Please share how this access benefits you. Your story matters.

Citation: Tsitsiklis, John N. and Xu, Yunjian. "Profit Loss in Cournot Oligopolies." Operations Research Letters 41, 4 (July 2013): 415-420 @ 2013 Elsevier B.V.

As Published: http://dx.doi.org/10.1016/j.orl.2013.04.012

Publisher: Elsevier

Persistent URL: http://hdl.handle.net/1721.1/110536

Version: Author's final manuscript: final author's manuscript post peer review, without publisher's formatting or copy editing

Terms of use: Creative Commons Attribution-NonCommercial-NoDerivs License 


\title{
Profit Loss in Cournot Oligopolies
}

\author{
John N. Tsitsiklis and Yunjian $\mathrm{Xu}^{1}$ \\ Laboratory or Information and Decision Systems, MIT, Cambridge, MA, 02139.
}

\section{Abstract}

We compare the aggregate profit achieved at a Cournot equilibrium to the maximum possible, which would be obtained if the suppliers were to collude. We establish a lower bound on the profit of Cournot equilibria in terms of a scalar parameter that captures qualitative properties of the inverse demand function and the number of suppliers (or the maximum of the suppliers' market shares). The lower bounds are tight when the inverse demand function is affine.

Keywords: Cournot oligopoly, Revenue management, Nash equilibrium

\section{Introduction}

\subsection{Background}

It is well known that oligopolists can increase their total profit by colluding to reduce their total output $[1,2]$. In this paper, we focus on the classical Cournot oligopoly model, and explore the potential gain in profits if the oligopolists were to collude or, equivalently, the reduction in profits due to competition. More concretely, we compare the aggregate profit earned at

\footnotetext{
${ }^{1}$ Corresponding author. Email address: xuyunjian@gmail.com.
} 
a Cournot equilibrium to the maximum possible profit, that is, the aggregate profit that would have been achieved if the suppliers were to collude.

Oligopolist profit loss due to competition has received some recent attention. Anderson and Renault [3] quantify the profit loss in Cournot oligopoly models with concave demand functions. However, most of their results focus on the relation between consumer surplus, producer surplus, and the aggregate social welfare achieved at a Cournot equilibrium, rather than on the relation between the aggregate profit achieved at a Cournot equilibrium and the maximum possible aggregate profit. Perakis and Sun [4] study supply chains with partial positive externalities and show that the profit loss at an equilibrium is at least $25 \%$ of the maximum profit.

Other recent works have reported various bounds on the profit loss at an equilibrium, for oligopoly models with affine demand functions. For a differentiated oligopoly model, Farahat and Perakis [5] establish lower and upper bounds on the profit loss at an equilibrium of price (Bertrand) competition. Closer to the present paper, Kluberg and Perakis [6] compare the aggregate profit earned by the suppliers under Cournot competition to the corresponding maximum possible, for the case where suppliers produce multiple differentiated products and the demand is an affine function of the price. However, one of their key assumptions (the invertibility of matrix $\mathbf{B}$ in the inverse demand function) does not hold in the Cournot model studied in this paper, even for the special case of affine inverse demand functions.

\subsection{Our contribution}

In this paper, we study the profit loss in a classical Cournot oligopoly model, for a broad class of nonincreasing inverse demand functions that result 
in concave revenue functions. In Section 3, we establish a lower bound of the form $f^{P}(c / d, N)$ on the profit ratio of a Cournot equilibrium (the ratio of the aggregate profit earned at the equilibrium to the maximum possible). Here, $f^{P}$ is a function given in closed form, $c / d$ is a scalar that captures a qualitative property of the inverse demand function, and $N$ is the number of suppliers. We also derive a profit ratio lower bound, of the form $g^{P}(c / d, r)$, which does not depend on the number of suppliers, but on the market share of the largest supplier at equilibrium, $r$. For Cournot oligopolies with affine inverse demand functions, we have $c / d=1$, and our lower bounds are tight. More generally, the scalar $c / d$ can be viewed as a measure of nonlinearity of the inverse demand function. As the parameter $c / d$ goes to infinity, the lower bounds converge to zero and arbitrarily high profit losses are possible.

Our results allow us to lower bound the profit ratio of Cournot equilibria for a large class of Cournot oligopoly models in terms of qualitative properties of the inverse demand function, without having to restrict to the special case of affine demand functions, and without having to calculate the equilibrium and the profit-maximizing output. In Section 4 we apply the derived lower bounds to various commonly encountered inverse demand functions. Our profit ratio lower bounds naturally provide upper bounds on the profit increase (in percentage terms) resulting from the formation of a cartel, which is one of the main incentives that drive oligopolists to collude.

This paper can be viewed as complementary to [11], which compares the social welfare at a Cournot equilibrium to the maximum possible social welfare. Although some of the questions and techniques in that paper are similar as here, the underlying assumptions, technical details, and results are 
quite different.

\section{Formulation and Preliminaries}

In this section, we first define the Cournot oligopoly model that we study in this paper, and introduce several main assumptions that we will be working with. We also show that profit ratio lower bounds can be obtained by restricting to linear cost functions and a special class of piecewise linear inverse demand functions. Our exposition here follows [11].

We consider a market for a single homogeneous good with inverse demand function $p:[0, \infty) \rightarrow[0, \infty)$ and $N$ suppliers. Supplier $n \in\{1,2, \ldots, N\}$ has a cost function $C_{n}:[0, \infty) \rightarrow[0, \infty)$. Each supplier $n$ chooses a nonnegative real number $x_{n}$, which is the amount of the good to be supplied by her. The strategy profile $\mathbf{x}=\left(x_{1}, x_{2}, \ldots, x_{N}\right)$ results in a total supply denoted by $X=\sum_{n=1}^{N} x_{n}$, and a corresponding market price $p(X)$. Supplier $n$ 's payoff is

$$
\pi_{n}\left(x_{n}, \mathbf{x}_{-n}\right)=x_{n} p(X)-C_{n}\left(x_{n}\right)
$$

where we have used the standard notation $\mathbf{x}_{-n}$ to indicate the vector $\mathbf{x}$ with the component $x_{n}$ omitted. A Cournot equilibrium is defined as a Nash equilibrium associated with the above defined payoff functions. For a function defined on a domain $[0, Q]$, the derivatives at the endpoints 0 and $Q$ are defined as right and left derivatives, respectively.

Assumption 1. For each $n$, the cost function $C_{n}:[0, \infty) \rightarrow[0, \infty)$ is convex, continuous, nondecreasing, and continuously differentiable. Furthermore, $C_{n}(0)=0$. 
Assumption 2. The inverse demand function $p:[0, \infty) \rightarrow[0, \infty)$ is continuous, nonnegative, and nonincreasing, with $p(0)>0$. Its right derivative at 0 exists and at every $q>0$, its left and right derivatives also exist.

Note that some parts of our assumptions are redundant, but are included for easy reference. For example, if $C_{n}(\cdot)$ is convex and nonnegative, with $C_{n}(0)=0$, then it is automatically continuous and nondecreasing.

The maximum possible profit earned by all suppliers (if they collude) is an optimal solution to the optimization problem,

$$
\begin{array}{ll}
\text { maximize } & p(X) \cdot X-\sum_{n=1}^{N} C_{n}\left(x_{n}\right) \\
\text { subject to } & x_{n} \geq 0, \quad n=1, \ldots, N, N
\end{array}
$$

where $X=\sum_{n=1}^{N} x_{n}$. We use $\mathbf{x}^{P}=\left(x_{1}^{P}, \ldots, x_{N}^{P}\right)$ to denote an optimal solution to (1), and let $X^{P}=\sum_{n=1}^{N} x_{n}^{P}$. We will refer to an optimal solution to (1) as a monopoly output. For a model with a nonincreasing continuous inverse demand function and continuous convex cost functions, the following assumption guarantees the existence of an optimal solution to (1), because it essentially restricts the optimization to a compact set of vectors $\mathbf{x}$ for which $x_{n} \leq R$, for all $n$.

Assumption 3. There exists some $R>0$ such that $p(R) \leq \min _{n}\left\{C_{n}^{\prime}(0)\right\}$.

Under Assumptions 1-3, there must exist an optimal solution to (1). Note however that there may exist multiple optimal solutions to (1), associated with different prices. We define $\mathcal{P}$ as the set of prices resulting from monopoly outputs. The following assumption guarantees that the objective function in (1) is concave on the interval where it is positive. 
Assumption 4. On the interval where $p(\cdot)$ is positive, the function $p(q) q$ is concave in $q$.

Assumption 4 is fairly common (cf. Section 4.1 of [7]), and implies that the best reply of each supplier is decreasing in the aggregate output of the other suppliers. Note that since $p(\cdot)$ is nonincreasing, any concave inverse demand function satisfies Assumption 4. Furthermore, many convex inverse demand functions, e.g., those of the form [8, 9],

$$
p(q)=\max \left\{\alpha-\beta q^{\delta}, 0\right\}, \quad \alpha, \beta, \delta>0,
$$

satisfy Assumption 4.

Under Assumptions 1, 2, and 4, the objective function in (1) is concave on the interval where it is positive. Hence, we have the following necessary and sufficient conditions for a vector $\mathbf{x}^{P}$ with $p\left(X^{P}\right)>0$ to maximize the aggregate profit:

$$
\left\{\begin{array}{l}
C_{n}^{\prime}\left(x_{n}^{P}\right) \leq p\left(X^{P}\right)+\partial_{-} p\left(X^{P}\right) \cdot X^{P}, \quad \text { if } x_{n}>0 \\
C_{n}^{\prime}\left(x_{n}^{P}\right) \geq p\left(X^{P}\right)+\partial_{+} p\left(X^{P}\right) \cdot X^{P}
\end{array}\right.
$$

where we use the notation $\partial_{+} p$ and $\partial_{-} p$ to denote the right and left directional derivative of $p$, respectively.

Similarly, under Assumptions 1 and 2, if $\mathbf{x}$ is a Cournot equilibrium, then

$$
\begin{aligned}
& C_{n}^{\prime}\left(x_{n}\right) \leq p(X)+x_{n} \cdot \partial_{-} p(X), \quad \text { if } x_{n}>0, \\
& C_{n}^{\prime}\left(x_{n}\right) \geq p(X)+x_{n} \cdot \partial_{+} p(X),
\end{aligned}
$$

where again $X=\sum_{n=1}^{N} x_{n}$. Note, however, that in the absence of further assumptions, the payoff of supplier $n$ need not be a concave function of $x_{n}$ and 
these conditions are, in general, not sufficient. We say that a nonnegative vector $\mathbf{x}$ is a Cournot candidate if it satisfies the necessary conditions (4)-(5). Thus, the set of Cournot equilibria is always a subset of the set of Cournot candidates.

As shown in [10], if $p(0)>\min _{n}\left\{C_{n}^{\prime}(0)\right\}$, then the aggregate supply at a Cournot equilibrium is positive; see Proposition 4 in [11] for a slight generalization to Cournot candidates. If on the other hand $p(0) \leq \min _{n}\left\{C_{n}^{\prime}(0)\right\}$, then the model is uninteresting, because no supplier has an incentive to produce and the optimal social welfare is zero. This motivates the assumption that follows.

Assumption 5. The price at zero supply is larger than the minimum marginal cost of the suppliers, i.e.,

$$
p(0)>\min _{n}\left\{C_{n}^{\prime}(0)\right\}
$$

Given a nonnegative vector $\mathbf{x}$, we define its profit ratio $\eta(\mathbf{x})$, by

$$
\eta(\mathbf{x})=\frac{X p(X)-\sum_{n=1}^{N} C_{n}\left(x_{n}\right)}{X^{P} p\left(X^{P}\right)-\sum_{n=1}^{N} C_{n}\left(x_{n}^{P}\right)},
$$

where $\left(x_{1}^{P}, \ldots, x_{N}^{P}\right)$ is an optimal solution to the optimization problem (1). Under Assumptions 1-5, the ratio is well defined, because the denominator is positive. It is easy to see that a Cournot candidate yields a nonnegative profit, and therefore its profit ratio is nonnegative. Due to space limitations, the proofs of the next three propositions are omitted, and can be found in $[12]$.

Proposition 1. Suppose that Assumptions 1-5 hold. Let $\mathbf{x}$ be a Cournot candidate. If $p(X) \notin \mathcal{P}$, then for any optimal solution $\mathbf{x}^{P}$ to (1), we have $X>X^{P}$. 
Proposition 2. Suppose that Assumptions 1-5 hold. Let $\mathbf{x}$ be a Cournot candidate. If $p(X) \in \mathcal{P}$ and $p^{\prime}(X)$ exists, then $\eta(\mathbf{x})=1$.

It can be shown that if the inverse demand function is convex, then $p^{\prime}(X)$ exists for any Cournot candidate $\mathbf{x}$ (cf. Proposition 3 in [11]). On the other hand, for a model satisfying Assumptions 1-5, if the inverse demand function is not differentiable at $X$, then a Cournot equilibrium $\mathbf{x}$ may yield arbitrarily large profit loss, even if $X=X^{P}$, and $N$ is held fixed (cf. Example 3 in [12]).

Proposition 3. Suppose that Assumptions 1-5 hold. Let $\mathbf{x}$ be a Cournot candidate that is not an optimal solution to (1), and let $\alpha_{n}=C_{n}^{\prime}\left(x_{n}\right)$. Consider a modified model in which we replace the cost function of each supplier $n$ by a new function $\bar{C}_{n}(\cdot)$, defined by

$$
\bar{C}_{n}(x)=\alpha_{n} x, \quad \forall x \geq 0 .
$$

Then, for the modified model, Assumptions 1-5 still hold, the vector $\mathbf{x}$ is a Cournot candidate and its profit ratio, denoted by $\bar{\eta}(\mathbf{x})$, satisfies $0 \leq \bar{\eta}(\mathbf{x}) \leq$ $\eta(\mathbf{x})$.

Note that Proposition 3 applies to all Cournot equilibria that do not maximize the aggregate profit.

Proposition 4. Let $\mathbf{x}$ and $\mathbf{x}^{P}$ be a Cournot candidate and a monopoly output, respectively. Suppose that Assumptions 1-5 hold, $p^{\prime}(X)$ exists, and that $p(X) \notin \mathcal{P}$. Let $c=\left|\left(p\left(X^{P}\right)-p(X)\right) /\left(X^{P}-X\right)\right|, d=\left|p^{\prime}(X)\right|$. Now consider a modified model in which the inverse demand function is replaced by the piecewise linear function $p^{0}(\cdot)$ defined by 


$$
p^{0}(q)= \begin{cases}-c(q-X)+p(X), & 0 \leq q \leq X, \\ \max \{0,-d(q-X)+p(X)\}, & X<q .\end{cases}
$$

Let $\eta^{0}(\mathbf{x})$ be the profit ratio of the vector $\mathbf{x}$ in the modified model. Then,

$$
\eta^{0}(\mathbf{x}) \leq \eta(\mathbf{x})
$$

According to Proposition 1, we have $X^{P}<X$, and therefore the constant $c$ and the function $p^{0}(q)$ in $(7)$ is well defined. The first segment of the piecewise linear function $p^{0}(\cdot)$ agrees with the inverse demand function $p(\cdot)$ at the two points $\left(X^{P}, p\left(X^{P}\right)\right)$ and $(X, p(X))$; the second segment is tangent to the inverse demand curve $p(\cdot)$ at the point $(X, p(X))$.

Proof. Since $p^{0}(X)=p(X)$, the aggregate profit earned at $\mathbf{x}$ is

$$
X p(X)-\sum_{n=1}^{N} C_{n}\left(x_{n}\right)
$$

in both the original and the modified model. Hence, we have

$$
\eta^{0}(\mathbf{x}) \leq \frac{X p(X)-\sum_{n=1}^{N} C_{n}\left(x_{n}\right)}{X^{P} p^{0}\left(X^{P}\right)-\sum_{n=1}^{N} C_{n}\left(x_{n}^{P}\right)}=\frac{X p(X)-\sum_{n=1}^{N} C_{n}\left(x_{n}\right)}{X^{P} p\left(X^{P}\right)-\sum_{n=1}^{N} C_{n}\left(x_{n}^{P}\right)}=\eta(\mathbf{x})
$$

where the inequality holds because the maximum total profit in the modified

model is at least $X^{P} p^{0}\left(X^{P}\right)-\sum_{n=1}^{N} C_{n}\left(x_{n}^{P}\right)$, and the next equality holds because $p^{0}\left(X^{P}\right)=p\left(X^{P}\right)$.

\section{Profit ratio lower bounds}

We fist establish a lower bound on the profit ratio of a Cournot candidate as a function of the scalar parameter $c / d$ and the maximum of the suppliers' 


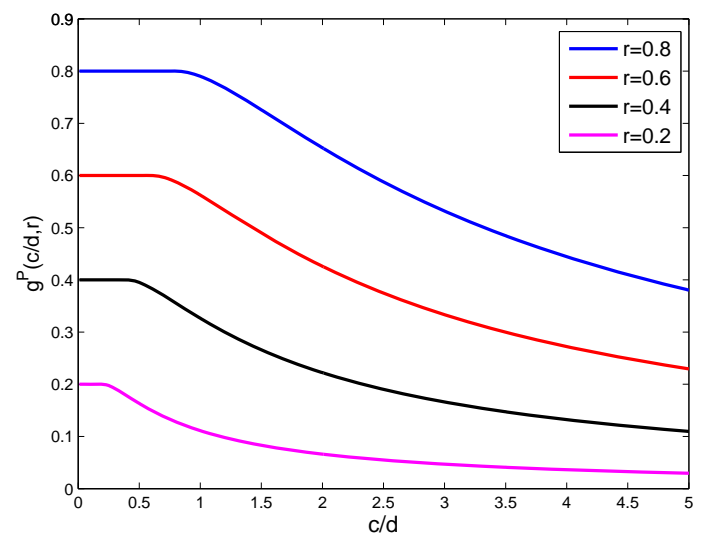

Figure 1: A lower bound on the profit ratio of a Cournot equilibrium as a function of the parameter $c / d$, for different values of the largest market share $r$.

market shares at an equilibrium (Theorem 1). Through a similar approach, we also provide a lower bound in terms of the scalar parameter $c / d$, and the number of suppliers (Theorem 2).

Theorem 1. Let $\mathbf{x}$ and $\mathbf{x}^{P}$ be a Cournot candidate and a monopoly output, respectively. Suppose that Assumptions 1-5 hold, $p^{\prime}(X)$ exists, and that $p(X)>0$. Let $c=\left|\left(p\left(X^{P}\right)-p(X)\right) /\left(X^{P}-X\right)\right|$ and $d=\left|p^{\prime}(X)\right|$.

(a) If $p(X) \in \mathcal{P}$, then $\eta(\mathbf{x})=1$;

(b) If $p(X) \notin \mathcal{P}$, then $N \geq 2$ and $p^{\prime}(X)<0$. We have $\eta(\mathbf{x}) \geq g^{P}(\bar{c}, r)$, where $\bar{c}=c / d, r$ is the maximum of the suppliers' market shares, i.e., $r=\max _{n}\left\{x_{n} / X\right\}$, and

$$
g^{P}(\bar{c}, r)= \begin{cases}r, & \text { if } 0<\bar{c} \leq r<1, \\ \frac{4 \bar{c} r^{2}}{(\bar{c}+r)^{2}}, & \text { if } 0<r<\bar{c}\end{cases}
$$




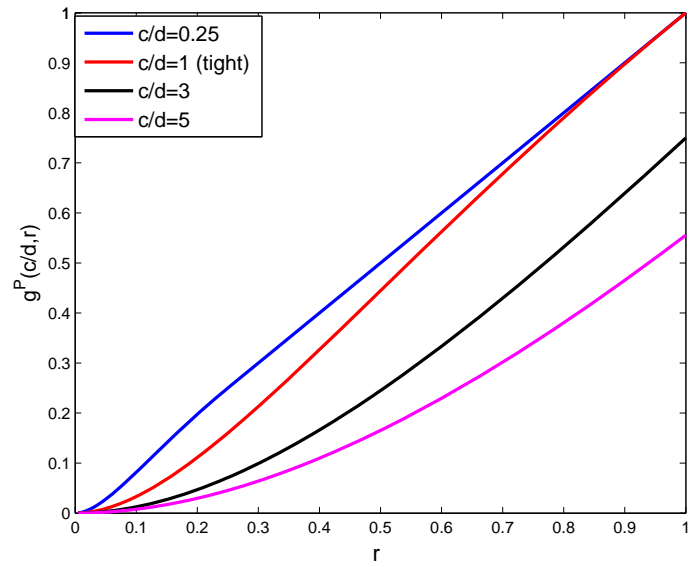

Figure 2: A lower bound on the profit ratio of a Cournot equilibrium as a function of the largest market share $r$, for different values of the parameter $c / d$.

(c) If $\bar{c}=1$ (in particular, if $p(\cdot)$ is affine), then $\eta(\mathbf{x}) \geq g^{P}(1, N)=$ $4 r^{2} /(1+r)^{2}$. Furthermore, the bound is tight. That is, for every $r \in$ $(0,1)$ and for every $\varepsilon>0$, there exists a model with $\bar{c}=1$ and a Cournot equilibrium whose profit ratio is no more than $4 r^{2} /(1+r)^{2}+\varepsilon$.

Theorem 1 is proved in Appendix A. The lower bounds are illustrated in Figs. 1 and 2. For a given $r$, the lower bound is nonincreasing in $\bar{c}=c / d$, and for a given $\bar{c}$, the lower bound increases with $r$. For affine inverse demand functions, we have $\bar{c}=1$ and the bound is tight (the red curve in Fig. 2).

Theorem 2. Suppose that the conditions in Theorem 1 hold, and that $p(X) \notin$ $\mathcal{P}$. We have: 


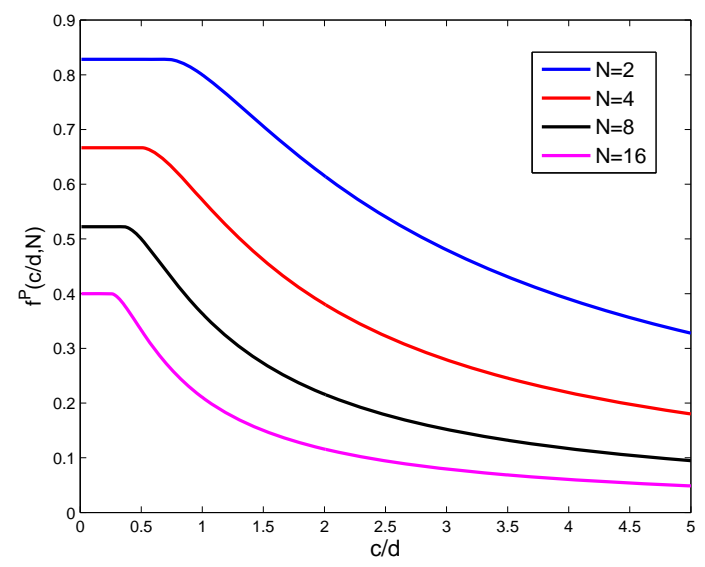

Figure 3: A lower bound on the profit ratio of a Cournot equilibrium as a function of the parameter $c / d$, for different values of $N$.

(a) $\eta(\mathbf{x}) \geq f^{P}(\bar{c}, N)$, where $\bar{c}=c / d$ and

$$
f^{P}(\bar{c}, N)= \begin{cases}\frac{N-1+(\sqrt{N}-1)^{2}}{\sqrt{N}(N-1)}, & \text { if } 0<\bar{c} \leq \sqrt{1 / N}, \\ \frac{4 \bar{c}^{3}(N-1)+4 \bar{c}(\bar{c}+1)^{2}}{\left(\bar{c}^{2} N+2 \bar{c}+1\right)^{2}}, & \text { if } \bar{c}>\sqrt{1 / N} .\end{cases}
$$

(b) If $\bar{c}=1$ (in particular, if $p(\cdot)$ is affine), then $\eta(\mathbf{x}) \geq f^{P}(1, N)=$ $4 /(N+3)$. Furthermore, the bound is tight, i.e., for any given $N \geq 2$, there exists a model with $\bar{c}=1$ and a Cournot equilibrium whose profit ratio is $4 /(N+3)$.

The proof of Theorem 2 can be found in Appendix A of [12]. It is straightforward to check that the function $f^{P}(\bar{c}, N)$ is strictly decreasing in $N$, as shown in Fig. 3. For any given $\bar{c}>0$, the lower bound, $f^{P}(\bar{c}, N)$, decreases to zero as the number of suppliers increases to infinity. Also, for any given $N$, the profit ratio lower bound is strictly decreasing in $\bar{c}$, over the interval 
$[\sqrt{1 / N}, \infty)$

\section{Corollaries and Applications}

Given the largest market share at an equilibrium (or the number of suppliers) and the inverse demand function $p(\cdot)$, the lower bounds derived in Theorem 1 (Theorem 2, respectively) require additional knowledge on the aggregate supply at the Cournot equilibrium and on the monopoly output, i.e., $X$ and $X^{P}$. In this section, we first establish profit ratio lower bounds that do not require the knowledge of $X$ and $X^{P}$, and then apply our results to calculate nontrivial quantitative profit ratio bounds for a class of inverse demand functions that have been considered in the economics literature.

Corollary 1. Suppose that Assumptions 1-5 hold, and that $p(\cdot)$ is concave and differentiable on the interval where it is positive. For every Cournot candidate $\mathbf{x}$ with $p(X)>0$, we have $\eta(\mathbf{x}) \geq \max \left\{f^{P}(1, N), g^{P}(1, r)\right\}$.

Corollary 2. Suppose that Assumptions 1-5 hold, and that $p(\cdot)$ is convex. Let

$s=\inf \left\{q \mid p(q)=\min _{n} C_{n}^{\prime}(0)\right\}, \quad t=\inf \left\{q \mid \min _{n} C_{n}^{\prime}(q) \geq p(q)+q \partial_{+} p(q)\right\}$.

If $\partial_{-} p(s)<0$, then the profit ratio of a Cournot candidate $\mathbf{x}$ with $p(X)>0$ is at least

$$
\max \left\{f^{P}\left(\partial_{+} p(t) / \partial_{-} p(s), N\right), g^{P}\left(\partial_{+} p(t) / \partial_{-} p(s), r\right)\right\}
$$

Note that under Assumption 3, the existence of the real numbers defined in (10) is guaranteed, and $t \leq s$. The proof of the preceding two corollaries 
can be found in [12]. We also note that if there exists a "best" supplier $n$ such that $C_{n}^{\prime}(x) \leq C_{m}^{\prime}(x)$, for any other supplier $m$ and any $x>0$, then the parameters $s$ and $t$ depend only on $p(\cdot)$ and $C_{n}^{\prime}(\cdot)$. In the following three examples, we apply Corollary 2 to three forms of convex inverse demand functions that appear in the economics literature.

Example 1. Suppose that Assumption 1, 3, and 5 hold. There are $N \geq 2$ suppliers. There exists a best supplier, the cost function of which is linear with a slope $\chi \geq 0$. Consider inverse demand functions of the form in Eq. (2), as in $[8,9]$,

$$
p(q)=\max \left\{\alpha-\beta q^{\delta}, 0\right\}, \quad \alpha, \beta, \delta>0 .
$$

It is not hard to see that Assumption 2 holds. Assumption 5 implies that $\alpha>\chi$. Since

$$
\frac{d^{2}(q p(q))}{d q^{2}} \leq p^{\prime}(q)+q p^{\prime \prime}(q)=-\beta \delta q^{\delta-1}-\beta \delta(\delta-1) q^{\delta-1}=-\beta \delta^{2} q^{\delta-1} \leq 0,
$$

we see that Assumption 4 holds. Through a simple calculation we have

$$
s=\left(\frac{\alpha-\chi}{\beta}\right)^{1 / \delta}, \quad t=\left(\frac{\alpha-\chi}{\beta(\delta+1)}\right)^{1 / \delta} .
$$

We also have

$$
\frac{p^{\prime}(t)}{p^{\prime}(s)}=\frac{-\beta \delta t^{\delta-1}}{-\beta \delta s^{\delta-1}}=(\delta+1)^{\frac{1-\delta}{\delta}} .
$$

From Corollary 2 we know that for every Cournot equilibrium $\mathbf{x}$ with $p(X)>$ 0 ,

$$
\eta(\mathbf{x}) \geq \max \left\{f^{P}\left((\delta+1)^{\frac{1-\delta}{\delta}}, N\right), g^{P}\left((\delta+1)^{\frac{1-\delta}{\delta}}, r\right)\right\} .
$$

We now argue that the preceding lower bound holds even without the assumption that there is a best supplier associated with a linear cost function. 
From Proposition 3, the profit ratio of any Cournot equilibrium $\mathbf{x}$ will not increase if the cost function of each supplier $n$ is replaced by

$$
\bar{C}_{n}(x)=C_{n}^{\prime}\left(x_{n}\right) x, \quad \forall x \geq 0 .
$$

Let $c=\min _{n}\left\{C_{n}^{\prime}\left(x_{n}\right)\right\}$. Since the profit ratio lower bound in (11) holds for the modified model with linear cost functions, it applies whenever the inverse demand function is of the form (2).

\section{Conclusion}

For Cournot oligopoly models with concave revenue functions, results such as those provided in Theorem 1 (or Theorem 2) show that the profit ratio at a Cournot equilibrium can be lower bounded by a function of the largest market share at the equilibrium $r$ (or the number of suppliers $N$ ), and a scalar parameter that captures quantitative properties of the inverse demand function. For the important special class of Cournot models with affine inverse demand functions, our profit ratio lower bounds, $4 r^{2} /(1+r)^{2}$ and $4 /(N+3)$, are tight.

\section{Acknowledgement}

This research was supported in part by the National Science Foundation under grant CMMI-0856063 and by a Graduate Fellowship from Shell.

\section{References}

[1] E. Chamberlin, Duopoly: Value where sellers are few, Q. J. Econ. 44(1929) 63-100. 
[2] J.W. Friedman, A non-cooperative equilibrium for supergames, Rev. Econ. Stud. 28(1971) 1-12.

[3] S. Anderson, R. Renault, Efficiency and surplus bounds in Cournot competition, J. Econ. Theory 113(2003) 253-264.

[4] G. Perakis, W. Sun, Price of anarchy for supply chains with partial positive externalities, Oper. Res. Lett. 40(2012) 78-83.

[5] A. Farahat, G. Perakis, Profit loss in differentiated oligopolies, Oper. Res. Lett. 37(2009) 43-46.

[6] J. Kluberg, G. Perakis, Generalized quantity competition for multiple products and loss of efficiency, Proc. Allerton Conf. Comm., Control, Comput., Monticello, IL, 2008.

[7] X. Vives, Oligopoly Pricing: Old Ideas and New Tools, MIT Press, Cambridge, MA, USA, 2001.

[8] J. Bulow, P. Pfleiderer, A note on the effect of cost changes on prices, J. Polit. Econ. 91(1983) 182-185.

[9] L.C. Corchon, Welfare losses under Cournot competition, Int. J. Ind. Organ. 26(2008) 1120-1131.

[10] J.W. Friedman, Oligopoly and the Theory of Games, North-Holland, Amsterdam. 1977.

[11] J.N. Tsitsiklis, Y. Xu, Efficiency loss in a Cournot oligopoly with convex market demand, submitted, 2011. http://arxiv.org/abs/1203.6675 
[12] J.N. Tsitsiklis, Y. Xu, Profit Loss in Cournot Oligopolies. http://www. mit.edu/ jnt/Papers/P-12-Cournot-profits-sub.pdf

[13] R. Tyagi, A characterization of retailer response to manufacturer trade deals, J. Mar. Res., 36(1999) 510-516. 


\section{Appendix A. Proof of Theorem 1}

According to Proposition 2, if $p(X) \in \mathcal{P}$, then the Cournot candidate's profit ratio must equal one.

To prove part (b), we assume that $p(X) \notin \mathcal{P}$. Note that we must have $N \geq 2$. This is because otherwise, a Cournot candidate $\mathbf{x}$ in a model with $N=1$ would satisfy the conditions (3). Since also $p(X)>0$, it would follow that the Cournot candidate maximizes the aggregate profit and $p(X) \in \mathcal{P}$, a contradiction. We also note that since $X>0, r$ is well defined. If $r=1$, then the Cournot candidate satisfies conditions (3), and therefore maximizes the aggregate profit. Hence, we have $r \in(0,1)$.

If $p^{\prime}(X)=0$, the necessary conditions (4)-(5) imply the conditions in (3). Since $p(X)>0$, the conditions in (3) are sufficient for $\mathbf{x}$ to maximize the aggregate profit. But since $p(X) \notin \mathcal{P}$, this cannot be the case and we must have $p^{\prime}(X)<0$ and $d>0$.

Without loss of generality, let supplier 1 have the largest market share, i.e., $x_{1}=\max _{n}\left\{x_{n}\right\}$ and $r=x_{1} / X$. According to Proposition 3 , the vector $\mathbf{x}$ remains a Cournot candidate in the modified model with linear cost functions, and Assumptions 1-5 still hold. Therefore, to lower bound the worst case profit ratio for Cournot candidates, we only need to derive a lower bound for the profit ratio of Cournot candidates for the case of linear cost functions. We therefore assume that $C_{n}\left(x_{n}\right)=\alpha_{n} x_{n}$ for each $n$. From the conditions in (4)-(5), it is straightforward to see that $\alpha_{1}=\min _{n}\left\{\alpha_{n}\right\}$.

Since $p(X) \neq p\left(X^{P}\right)$, we have that $c>0$. For notational convenience, we let $y=p(X)$ throughout the proof. We will prove the theorem by considering separately the cases where $\alpha_{1}=0$ and $\alpha_{1}>0$. 


\section{The case $\alpha_{1}=0$}

Let $\mathbf{x}$ be a Cournot candidate in the original model, with linear cost functions and the inverse demand function $p(\cdot)$. Since $p^{\prime}(X)<0$, the conditions in (4) and (5) yield $y>0, x_{1}=y / d$ and $r=y / d X$. We therefore have

$$
0 \leq \sum_{n=2}^{N} x_{n}=X-\frac{y}{d}
$$

From Proposition 4, the profit ratio of the vector $\mathbf{x}$ in the modified model (with a piecewise linear inverse demand function of the form in $(7)$ ), $\eta^{0}(\mathbf{x})$, cannot be more than its profit ratio in the original model, $\eta(\mathbf{x})$. Hence, to prove part (b), it suffices to show that $\eta^{0}(\mathbf{x}) \geq g^{P}(\bar{c}, r)$. For the modified model, the maximum aggregate profit is the optimal value of the following optimization problem,

$$
\begin{aligned}
& \text { maximize } q p^{0}(q) \\
& \text { subject to } q \geq 0 .
\end{aligned}
$$

Through a simple calculation we have:

(i) If $c X \geq y$, then the maximum aggregate profit is $(c X+y)^{2} /(4 c)$, achieved at $q=(c X+y) /(2 c)$.

(ii) If $c X \leq y$, then the maximum aggregate profit is $X y$, achieved at $q=X$.

Note that for $n=1$ we have $\alpha_{n} x_{n}=0$. For $n \geq 2$, whenever $x_{n}>0$, from conditions (4) and (5) we have $\alpha_{n}=y-x_{n} d$ and $\alpha_{n} x_{n}=\left(y-x_{n} d\right) x_{n}$. 
Therefore, in the modified model, the aggregate profit achieved at $\mathbf{x}$ is

$$
\begin{aligned}
X p(X)-\sum_{n=1}^{N} \alpha_{n} x_{n} & =X y-\sum_{n=2}^{N}\left(y-x_{n} d\right) x_{n} \\
& \geq X y-y \sum_{n=2}^{N} x_{n}+\frac{(X d-y)^{2}}{(N-1) d} \\
& =X y-y(X-y / d)+\frac{(X d-y)^{2}}{(N-1) d} \\
& =\frac{y^{2}}{d}+\frac{(X d-y)^{2}}{(N-1) d},
\end{aligned}
$$

where the inequality is true because $\sum_{n=2}^{N} x_{n}^{2}$ is minimized when $x_{2}=x_{3}=$ $\cdots=x_{N}$, subject to the constraint in (A.1). For the case $c X \geq y$, since the maximum aggregate profit is $(c X+y)^{2} / 4 c$, we have

$$
\eta^{0}(\mathbf{x}) \geq \frac{y^{2} / d+(X d-y)^{2} /((N-1) d)}{(c X+y)^{2} / 4 c} .
$$

Note that $c, d$ and $y$ are positive. Substituting $r=y / d X$ and $\bar{c}=c / d$ in (A.3), we have

$$
\eta^{0}(\mathbf{x}) \geq \frac{4 \bar{c}^{2} r^{2}+\frac{4(\bar{c}-\bar{c} r)^{2}}{N-1}}{\bar{c}(\bar{c}+r)^{2}} \geq \frac{4 \bar{c}^{2} r^{2}}{\bar{c}(\bar{c}+r)^{2}}=\frac{4 \bar{c} r^{2}}{(\bar{c}+r)^{2}}, \quad 0 \leq r \leq \min \{\bar{c}, 1\},
$$

where the constraint $r \leq \bar{c}$ follows from $c X \geq y$.

For the case $c X \leq y$, we have $r \geq \bar{c}$, and the maximum aggregate profit is $X y$. From (A.2), we have

$$
\eta^{0}(\mathbf{x}) \geq \frac{\frac{y^{2}}{d}+\frac{(X d-y)^{2}}{(N-1) d}}{X y}=\frac{\bar{c}^{2} r^{2}(N-1)+(\bar{c}-\bar{c} r)^{2}}{\bar{c}^{2} r(N-1)} \geq r, \quad 0<\bar{c} \leq r<1 .
$$




\section{Tightness}

Given some $r \in(0,1)$, consider a model with $N \geq\lceil 1 / r\rceil+1$, and an affine inverse demand function $p^{0}(\cdot)$ of the form in (7), where $c / d=1$ and $r d X=y$. The cost of supplier 1 is identically zero and

$$
C_{n}(x)=\left(y-\frac{d}{N-1}(X-r X)\right) x, \quad n=2, \ldots, N .
$$

It is not hard to see that the vector with components

$$
x_{1}=r X, \quad x_{n}=\frac{1}{N-1}(X-r X), \quad n=2, \ldots, N
$$

satisfies the conditions (4)-(5). It is straightforward to check that $\mathbf{x}$ is a Cournot equilibrium. The maximum total profit is $(c X+y)^{2} / 4 c$, and is achieved at the monopoly output $\mathbf{x}^{P}=((c X+y) / 2 c, 0, \ldots, 0)$. On the other

hand, the aggregate profit achieved at $\mathbf{x}$ is given on the right-hand side of (A.2). We have

$$
\eta^{0}(\mathbf{x})=\frac{y^{2} / d+(X d-y)^{2} /((N-1) d)}{(c X+y)^{2} / 4 c}=\frac{4 r^{2}+4(1-r)^{2} /(N-1)}{(1+r)^{2}},
$$

and as the number of suppliers increases to infinity, the profit ratio of the Cournot equilibrium converges to $4 r^{2} /(1+r)^{2}$.

The case $\alpha_{1}>0$

By rescaling the cost coefficients and permuting the supplier indices, we can assume that $\min _{n}\left\{\alpha_{n}\right\}=\alpha_{1}=1$. It is straightforward to check (from the conditions in Eq. (4)-(5)) that $y \geq 1$ and $x_{1}=(y-1) / d$, i.e., $r=(y-1) / d X$. The desired results in (8) can be proved through an approach similar to the case that $\alpha_{1}=0$, by replacing $y$ with $y-1$. 